\title{
Relação entre condição gengival e fatores sociodemográficos de adolescentes residentes em uma região brasileira
}

\author{
The relationship between gingival condition and \\ socio-demographic factors of adolescents living in a Brazilian region
}

Emilio Prado Fonseca ${ }^{1}$

Efigênia Ferreira e Ferreira ${ }^{1}$

Mauro Henrique Nogueira Guimarães Abreu ${ }^{1}$

Andréa Clemente Palmier ${ }^{1}$

Andréa Maria Duarte Vargas ${ }^{1}$

${ }^{1}$ Departamento de Odontologia Social e Preventiva, Faculdade de Odontologia, Universidade Federal de Minas Gerais. Av. Presidente Antônio Carlos 6627, Pampulha. 31270-010 Belo Horizonte MG Brasil. emiliopraddo@ig.com.br

\begin{abstract}
The scope of this study is to correlate the gingival condition and sociodemographic status of adolescents living in an economically disadvantaged Brazilian region. The survey was conducted with a random sample of 450 adolescents in 13 cities selected in the Jequitinhonha Valley (State of Minas Gerais). The gingival condition was evaluated using a calibrated examiner (Kappa $\geq$ 0.85). The oral exam was based on the Community Periodontal Index (CPI). The frequencies of individuals were calculated and sextants subsequently assessed using Multiple Correspondence Analysis (ANACOR) to reveal the relationship between CPI and sociodemographic characteristics. The results showed that: 16 (3.6\%) were healthy; $235(52.2 \%)$ had gingival bleeding; 36 (8\%) had dental calculus and 163 (36.2\%) had both bleeding and calculus. ANACOR identified two groups with similarities in relation to periodontal disease. Group one featured 19-year-olds with healthy CPI who work and have higher family income. Group two included 15- and 16-year-olds of both sexes with CPI and gingival bleeding in elementary school with lower family income, who declared themselves to be of mixed or Afro-descendant race. The presence of gingivitis was related to the socioeconomic and demographic characteristics of adolescents in the region.

Key words Adolescent, Epidemiology, Socioeconomic factors, Periodontal index
\end{abstract}

Resumo O objetivo deste artigo é relacionar as condições gengival e socioeconômica e distribuição demográfica de adolescentes residentes em uma região desfavorecida brasileira. O estudo transversal foi realizado com amostra probabilística de 450 adolescentes (15-19 anos) em 13 municípios sorteados no Vale do Jequitinhonha $(M G)$. A condição gengival foi avaliada por um examinador calibrado (Kappa $\geq 0,85)$ e baseado Indice Periodontal Comunitário (CPI). Foram calculadas as frequências para indivíduos e sextantes com posterior Análise de Correspondência Múltipla (Anacor) que explorou relações entre o CPI e as características sociodemográficas. Como resultados: 16 (3,6\%) apresentaram-se sem alterações gengivais, 235 (52,2\%) com sangramento, $36(8,0 \%)$ com cálculo dental e 163 (36,2\%) com sangramento e cálculo. A Anacor identificou dois grupos que guardavam semelhanças entre si em relação à doença periodontal. O grupo 1 reuniu os adolescentes com CPI sadio, 19 anos de idade, que trabalham e têm maior renda familiar. No grupo de CPI com sangramento, tem-se os adolescentes com 15 e 16 anos de idade, de ambos os sexos, cursando o ensino fundamental, menor renda familiar e que se autodeclararam pardos ou negros. A gengivite foi relacionada às condiçães socioeconômicas e demográficas de adolescentes da região. Palavras-chave Adolescente, Epidemiologia, Fatores socioeconômicos, Índice periodontal 


\section{Introdução}

As doenças bucais constituem um grave problema da saúde pública brasileiro e de outros países ${ }^{1-4}$. Em especial as doenças periodontais que possuem significativas prevalências, refletem diretamente na qualidade de vida de indivíduos e estão associadas à perda dental ${ }^{1,4,5}$. Ou seja, o processo saúde/doença periodontal é dinâmico, em muitos casos reversível, sujeito a fatores de ordem individual, coletiva e que interagem entre si, sendo a placa bacteriana associada à má higiene oral e apontada como um dos principais fatores etiológicos dessa enfermidade ${ }^{4,5}$. Neste sentido, populações residentes em regiões menos favorecidas economicamente estão mais sujeitas a desenvolverem agravos, inclusive periodontais, em função da baixa renda familiar, menor acesso a bens e serviços públicos de saúde bucal ${ }^{1-3,6-11}$.

Estudos epidemiológicos periodontais apontam para altas prevalências de sangramento gengival e cálculo dental em diversos grupos populacionais e faixas etárias, inclusive em adolescentes $^{1,7,8-11}$. Em 2003 e $2010^{8,11}$ foram realizados estudos de base populacional sobre as condições de saúde bucal da população brasileira, inclusive de adolescentes na faixa etária de 15 a 19 anos, sendo que as regiões mais pobres apresentaram altas prevalências para sangramento gengival e cálculo dental. Outros estudos ${ }^{2,3,6,7,9,10,12}$ também demonstraram que as regiões mais pobres apresentaram maiores prevalências para sangramento gengival e cálculo dental. Entretanto, o comportamento das doenças periodontais em adolescentes, diante dos determinantes sociais da saúde desfavoráveis, ainda não estão suficientemente claros. Além de novos estudos possibilitarem a formulação de hipóteses explicativas ou da inclusão de novos fatores relacionados ao adoecimento de adolescentes ${ }^{13}$.

O objetivo deste estudo foi relacionar a condição gengival e fatores contextuais de adolescentes residentes em uma região economicamente desfavorecida brasileira através da Análise de Correspondência Múltipla (Anacor).

\section{Metodologia}

A mesorregião do Vale do Jequitinhonha está localizada ao Norte do Estado de Minas Gerais e é considerada uma das mais pobres do Brasil. Os indicadores socioeconômicos são muito des- favoráveis e a região possui baixo Índice de Desenvolvimento Humano (IDH) na maioria dos municípios, que também são de pequeno porte (até 2030 habitantes) ${ }^{14}$. A região também possui fragilidade no acesso à água tratada, à rede de esgoto e aos serviços de saúde ${ }^{14}$ (Figura 1).

Este estudo observacional do tipo transversal faz parte de um projeto maior e foi realizado em 52 municípios do Vale do Jequitinhonha em Minas Gerais, sobre condições de saúde bucal de adolescentes, no período de maio a agosto de 2010 e aprovado pelo Comitê de Ética da UFMG.

\section{População de Referência do Estudo}

A amostra foi composta por adolescentes, na faixa etária de 15 a 19 anos, que correspondem a aproximadamente $12 \%$ da população residente na região. A escolha dos adolescentes justifica-se por ser essa uma das faixas etárias preconizadas pela Organização Mundial de Saúde (OMS) para avaliação de saúde bucal. Em alguns municípios dessa região a implantação das equipes de saúde bucal é recente e a atenção muitas vezes ainda segue a ideia do sistema incremental que privilegia os escolares até 14 anos de idade. Portanto, os indivíduos que estão deixando a escola e entrando no mercado de trabalho podem estar excluídos das ações de saúde bucal no setor público e apresentar limitada utilização de atendimento odontológico.

O cálculo amostral foi obtido com base no de estimativa de proporção ${ }^{15} \mathrm{com}$ nível de significância de $95 \%$, erro admissível de 5\% e prevalência de 17,41\% (gengivite) segundo dados do SB Brasil $2003^{8}$. O cálculo amostral totalizou 221 adolescentes. O desenho do estudo utilizou amostra por conglomerados (cluster) em dois estágios: estágio um, os municípios, e estágio dois, os indivíduos. Esse tipo de amostragem altera a precisão das estimativas, já que estas dependem do grau de homogeneidade interna dos conglomerados $^{15}$. Ao se proceder essa técnica de amostragem perde-se a homogeneidade, e, portanto, um número mais elevado é requerido para compensar esse aspecto ${ }^{15}$. Essa correção pode ser efetuada de forma simplificada e conservadora, multiplicando-se o tamanho da amostra por dois. Esse procedimento é denominado efeito de delineamento ou efeito do desenho ${ }^{16}$. Acrescidos os $5 \%$ para compensar possíveis perdas, a amostra final totalizou a necessidade de inclusão de 464 adolescentes. Houve uma perda de 14 indivíduos $(3,01 \%)$. 
Figura 1. Localização geográfica da mesorregião do Vale do Jequitinhonha, Minas Geras, Brasil, 2012.

\section{Seleção dos municípios (Estágio 1)}

A mesorregião do Vale do Jequitinhonha é composta por 52 municípios ${ }^{14}$. Para contemplar a diversidade entre os municípios, a seleção dos mesmos foi feita considerando o porte populacional e o desempenho do serviço de saúde bucal de cada município. Com as duas variáveis foi elaborada uma matriz $3 \times 3$ e os municípios que se encontravam na diagonal principal foram selecionados para o estudo. Assim, foram formados três grupos: municípios com menor porte populacional e pior desempenho do serviço; municípios com médio porte populacional e médio desempenho do serviço; e municípios com maior porte populacional e melhor desempenho do serviço. Dessa forma, foram selecionados 13 municípios - unidade amostral primária. Considera-se importante avaliar a semelhança entre os municípios da amostra e os outros da região em relação aos critérios escolhidos e aos indicadores socioeconômicos. Quando comparados pelo teste de Mann-Whitney, não foram observados resultados estatisticamente significativos para as variáveis porte populacional $(\mathrm{p}=0,061)$ e desempenho do serviço $(p=0,431)$. As seguintes variáveis foram selecionadas para caracterizar socioeconomicamente os municípios: percentual de pessoas de 25 anos ou mais analfabetas $(\mathrm{p}=0,925)$, percentual de pessoas com mais de $50 \%$ da sua renda proveniente de transferências governamentais $(\mathrm{p}=0,533)$, IDH $(\mathrm{p}=0,719)$, Índice de Theil $(\mathrm{p}=0,069)$, Índice de Gini $(\mathrm{p}=$ $0,107)$, percentual de moradores com acesso à água tratada $(\mathrm{p}=4,79)$, percentual de moradores com acesso à rede geral de esgoto ou pluvial $(\mathrm{p}$ $=0,286)$, Índice de Necessidade em Saúde ( $\mathrm{p}=$ $4,39)$, Índice de Porte Econômico $(p=1,39)$ e FA $(\mathrm{p}=0,375)$. Portanto, não foi observada diferença entre municípios selecionados e não selecionados.

Considera-se, ao utilizar amostra complexa por conglomerado, probabilidades desiguais dos indivíduos ao comporem a amostra ${ }^{16,17}$. Neste caso, o método utilizado é a amostragem com Probabilidade Proporcional ao Tamanho (PPT), 
que é de fácil aplicação e reduz a variância entre as unidades de seleção ${ }^{17}$. Para a distribuição do total de participantes a ser incluído no estudo, em cada município foi calculada a proporcionalidade, de acordo com o total de adolescentes de cada um. Verificada a população de 15 a 19 anos de idade de cada município no DATA/SUS para o ano de 2010, foi utilizada a proporção da população nessa faixa etária correspondente de cada município. Posteriormente foi determinado o percentual da amostra para cada município, utilizando-se a mesma proporção.

\section{Seleção dos adolescentes (Estágio 2)}

Uma vez definidos os municípios e o número de indivíduos que seriam examinados e entrevistados em cada um deles, foi realizado o sorteio das unidades amostrais secundárias ${ }^{18}$. Como todos os municípios apresentavam menos de 50.000 habitantes, as unidades amostrais secundárias foram as quadras ${ }^{18}$. Todas as quadras urbanas foram numeradas e tiveram a mesma chance de sorteio. Foram excluídas do sorteio as quadras não habitadas (terrenos baldios, fábricas, hotéis e outros). Os 450 adolescentes foram selecionados de forma aleatória a partir de quadras sorteadas pelo programa Statistical Package for Social Sciences (SPSS), versão 18.0, para garantir chances iguais para cada quadra participar da amostra. As quadras sorteadas foram marcadas no mapa cartográfico do município. Para isso foi consultada a tabela contida no Manual do Coordenador do SB2000 que indicava o número de domicílios que deveriam ser visitados de acordo com o porte do município e a macrorregião ${ }^{18}$. O valor indicado na tabela deveria ser dividido pelo número médio de domicílios por quadra para obter o número de quadras a serem sorteadas.

A coleta de dados foi autorizada pelas secretarias de saúde municipal por meio da assinatura de um termo de anuência dos responsáveis pelo município, que foram enviados por meio eletrônico e posteriormente devolvidos assinados pelo correio.

O pesquisador examinador foi previamente treinado para obtenção de concordância e para permitir uma interpretação uniforme e consistente dos critérios (Kappa interexaminador = 0,887 e intraexaminador $=0,850$ ).

Participaram da coleta de dados os indivíduos que tinham entre 15 e 19 anos na data do exame, funcionalmente independentes, sem dificuldades cognitivas ou mentais e que aceitaram participar do estudo. Utilizou-se um questioná- rio socioeconômico baseado em instrumentos de dois estudos: SB Brasil $2003^{8}$ e Coorte de Pelo$\operatorname{tas}^{19}$ e exame clínico, ambos realizados no local de domicílio dos jovens. As variáveis contextuais analisadas foram: sexo, escolaridade, idade, atividade de trabalho, renda familiar e cor da pele, e o desfecho foi a presença de gengivite (sangramento à sondagem).

A equipe foi composta por um examinador e três anotadores (acadêmicos voluntários do curso de odontologia da Universidade Federal de Minas Gerais). Em alguns municípios a equipe contou com o auxílio dos Agentes Comunitários de Saúde para a localização das quadras sorteadas e apresentação aos moradores.

Para o registro dos dados periodontais foi utilizado o Índice Periodontal Comunitário (CPI) com algumas modificações ${ }^{18,20}$. Foram utilizados para cada sextante os seguintes códigos: $0=$ periodonto saudável; 1 = sangramento após sondagem; 2 = cálculo; 6 = sangramento após sondagem e cálculo; 5 = dente excluído; $9=$ não registrado. Os índices 3 e 4 do CPI (bolsas periodontais) não foram utilizados nesse estudo. Os exames para avaliar a condição periodontal foram realizados com iluminação natural, em local reservado do domicílio de forma a preservar a privacidade dos examinados. As normas de biossegurança foram obedecidas e a pesquisadora utilizou Equipamento de Proteção Individual (EPI) completo. Os instrumentos de coleta foram espelho clínico e sonda universal milimetrada, devidamente empacotados e esterilizados.

\section{Análise dos dados}

As variáveis contextuais analisadas foram: sexo, escolaridade, idade, atividade de trabalho, renda familiar e cor da pele autodeclarada.

Foi realizada uma análise descritiva das variáveis de estudo. A amostra também foi analisada por sextante e código CPI (sadio, sangramento, cálculo e sangramento + cálculo). Posteriormente, buscou-se explorar relações conjuntas entre os critérios do CPI avaliados e as variáveis contextuais através da Análise de Correspondência Múltipla (Anacor). Trata-se de uma técnica indicada para descrever matrizes com dados categóricos/ discretos com grande número de variáveis, com o objetivo de visualizar as relações mais importantes entre variáveis e categorias em um gráfico de pontos $^{21-23}$. Com essa técnica é possível interpretar as relações das categorias das variáveis "agrupando-as” por proximidade geométrica de modo 
a identificar perfis semelhantes para explicar a doença periodontal. O ponto de partida foi uma matriz, na qual, nas linhas temos os adolescentes e nas colunas as variáveis de interesse. No cruzamento entre linhas e colunas obtém-se um "perfil" definido do conjunto de dados ${ }^{21}$. Ao ignorar o desenho amostral na análise dos dados, podem ocorrer incorreções que comprometam os resultados, o teste de hipótese e as conclusões da pesquisa $^{17}$. O artigo é descritivo e a Anacor é uma metodologia de análise que não testa hipótese, mas relaciona variáveis. Portanto, a análise não é baseada em variância e desvio-padrão para cálculo do intervalo de confiança e teste de hipótese.

Para análise dos dados foram utilizados os programas Windows Excel ${ }^{\circledR}$ versão 2007 e Statistical Package for Social Sciences ${ }^{\circledR}$ (SPSS), versão 19.

\section{Resultados}

Foram examinados 450 adolescentes do grupo etário de 15 a 19 anos, dos quais 242 eram mulheres $(53,8 \%)$. A maioria se declarou parda $(63,12 \%)$, estudante $(76,9 \%)$, com atividade de trabalho $(62,89 \%)$ e vivendo em famílias cuja renda familiar era de até 3 salários mínimos (88\%) (Tabela 1).

Dos adolescentes, $16(3,6 \%)$ apresentaramse sem alterações gengivais, 232 (51,6\%) tinham sangramento, $38(8,4 \%)$ cálculo dental e 164 $(36,4 \%)$ sangramento e cálculo (Tabela 2).

De um total de 2700 sextantes, 791 (29,30\%) estavam sadios, 1392 (51,56\%) com sangramento, $94(3,48 \%)$ com cálculo e $422(15,63 \%)$ com sangramento e cálculo, e um perdido (0,03\%). Verificou-se que 49 (10,89\%) indivíduos tinham todos os sextantes com sangramento e 15 (3,33\%) apresentaram sangramento e cálculo em todos os sextantes. O sextante superior anterior foi o que apresentou menos alteração gengival; em relação à prevalência das alterações gengivais, o sextante superior posterior esquerdo apresentou maior prevalência de sangramento; o sextante anterior inferior, cálculo e o sextante superior esquerdo, sangramento e cálculo (Tabela 3).

A análise por correspondência permitiu identificar dois grupos distintos em relação à doença periodontal: o grupo 1 reuniu os adolescentes com periodonto sadio, 19 anos de idade, que trabalhavam e tinham maior renda familiar; no grupo 2 ficaram os adolescentes com sangramento gengival, idade de 15 e 16 anos, de ambos os sexos, cursando o ensino fundamental, com menor ren- da familiar e que se declararam pardos ou negros. Os adolescentes com idade entre 17 e 18 anos, renda familiar entre 1 e 3 salários, brancos, indígenas e cursando o ensino superior não relacionaram com o grupo sadio ou doente. (Figura 2).

Tabela 1. Características dos adolescentes segundo variáveis socioeconômicas e demográficas, Vale do Jequitinhonha (MG), 2010.

\begin{tabular}{lr}
\hline \multicolumn{1}{c}{ Variável } & $\mathbf{n}(\%)$ \\
\hline Sexo & \\
Masculino & $208(46,22)$ \\
Feminino & $242(53,78)$ \\
Atividade de trabalho & \\
Sim & $167(37,11)$ \\
Não & $283(62,89)$ \\
Idade em anos & \\
15 & $108(24)$ \\
16 & $112(24,89)$ \\
17 & $86(19,11)$ \\
18 & $75(16,67)$ \\
19 & $69(15,33)$ \\
Cor da pele autodeclarada & \\
Branca & $56(12,44)$ \\
Negra & $49(10,88)$ \\
Amarela & $17(3,77)$ \\
Parda & $284(63,12)$ \\
Indígena & $11(2,44)$ \\
Não respondeu & $33(7,34)$ \\
Escolaridade & \\
Ensino Fundamental & $169(37,55)$ \\
Ensino Médio/Técnico & $264(58,67)$ \\
Superior & $17(3,78)$ \\
Renda Familiar & \\
Até 1 salário mínimo & $160(35,55)$ \\
Entre 1 e 3 salários & $236(52,44)$ \\
Maior que 3 salários & $43(9,56)$ \\
Não informado & $11(2,45)$ \\
& \\
\hline &
\end{tabular}

"Salário mínimo utilizado como referência no estudo foi de R\$ 510,00. Critério padrão de Classificação Econômica - Critério Brasil 2008.

Tabela 2. Condições gengivais por sexo, 15-19 anos, Vale do Jequitinhonha, (MG), 2010.

\begin{tabular}{lrrr}
\hline & \multicolumn{2}{c}{ Sexo } & \\
\cline { 2 - 3 } & \multicolumn{1}{c}{ M } & \multicolumn{1}{c}{ F } & Total n (\%) \\
\hline Hígido & 8 & 8 & $16(3,55)$ \\
Sangramento & 92 & 140 & $232(51,56)$ \\
Cálculo & 22 & 16 & $38(8,44)$ \\
Sangramento/cálculo & 86 & 78 & $164(36,44)$ \\
Total & 208 & 242 & 450 \\
\hline
\end{tabular}




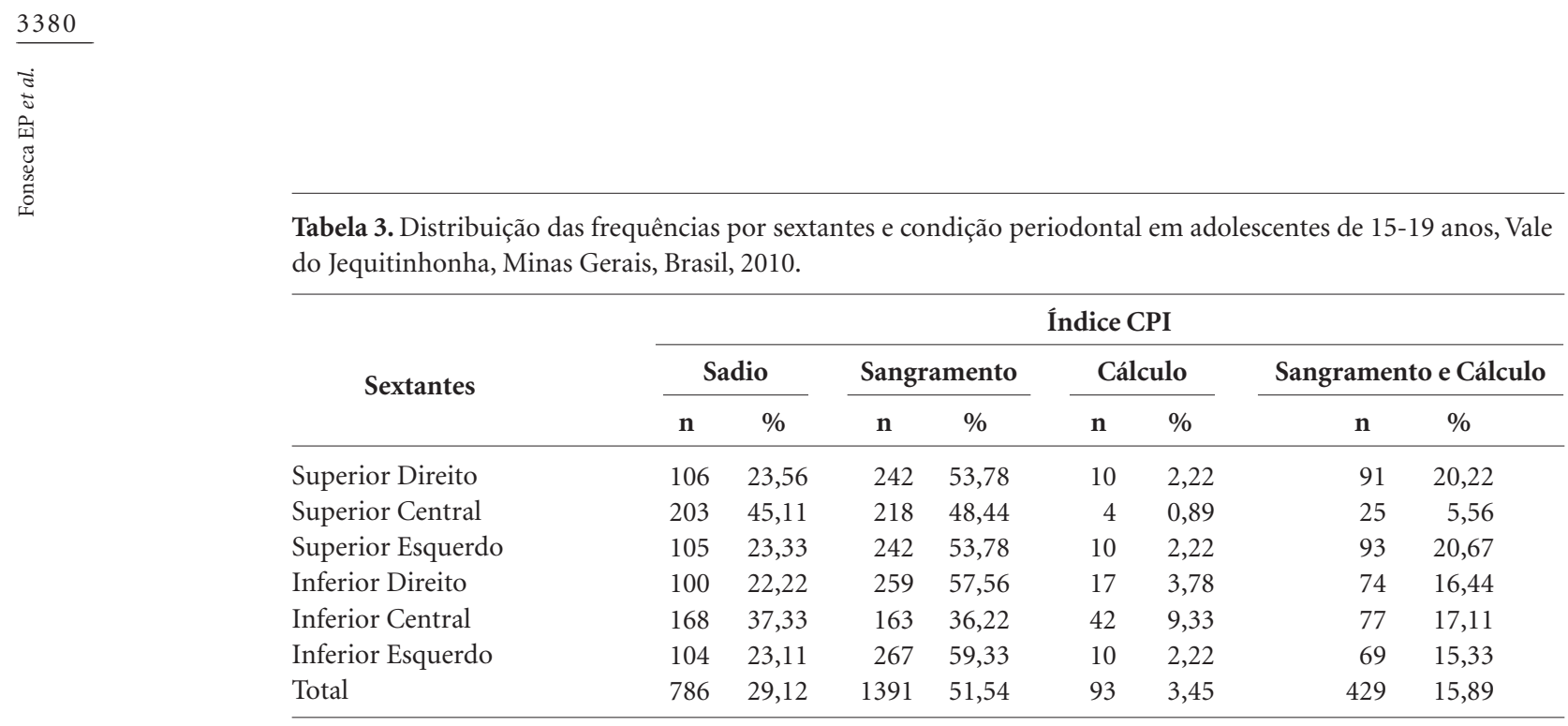

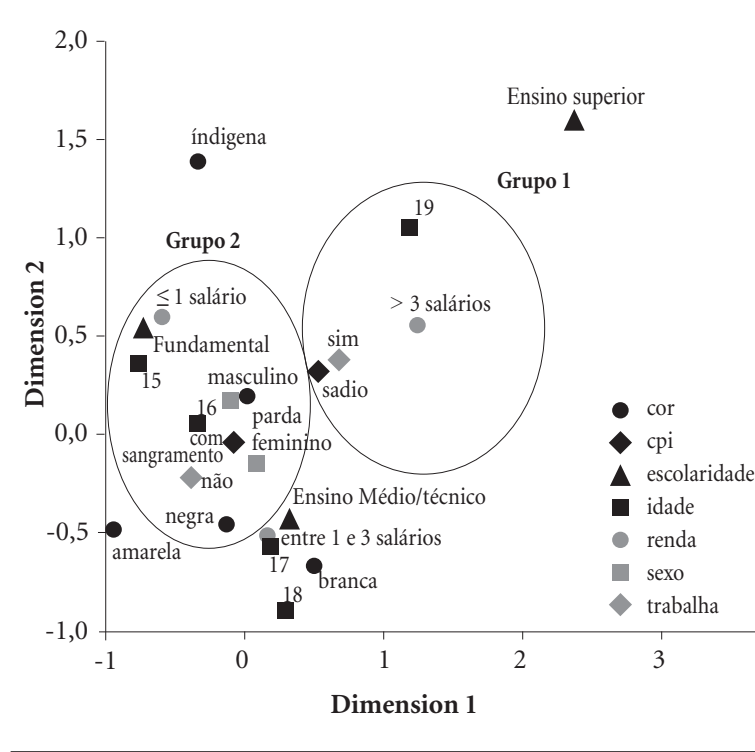

Figura 2. Categorias dos escores referentes ao CPI e as características socioeconômicas e demográficas resultantes da análise de correspondência para duas dimensões.

\section{Discussão}

Os inquéritos populacionais são importantes para o monitoramento da situação de saúde bucal das populações ${ }^{17}$. Sendo que um dos problemas bucais mais prevalentes na população é a gengivite. É possível de ser detectada com a presença de vermelhidão, tumefação gengival, tendência de sangramento após sondagem do sulco gengival $^{5}$ e representa o estágio reversível da doença periodontal. O cálculo dental é caracterizado como depósito mineralizado em superfícies dentárias, restaurações, implantes ou próteses que facilita o depósito de placa bacteriana ${ }^{5}$ e está relacionado com o autocuidado e o acesso ao serviço de saúde ${ }^{6}$. Conhecer as prevalências de sangramento gengival e cálculo dental na população de adolescentes é importante porque a presença destes sinais indica probabilidade de doença periodontal e de acesso ao serviço de saúde bucal em regiões desfavorecidas economicamente $e^{1-3,9,10}$. Mesmo que estes estudos sejam descritivos e realizados a nível local/regional, contemplam características e peculiaridades que as pesquisas epidemiológicas amplas não alcançam ${ }^{13}$. Além do mais, a localização geográfica de regiões e indivíduos em situação de maior vulnerabilidade constitui uma importante estratégia para o planejamento de ações de saúde bucal, permitem a reorientação e a alocação de recursos materiais, humanos e financeiros conforme a realidade da comunidade pesquisada e, consequentemente, melhoria no acesso aos serviços de saúde bucal ${ }^{9,17}$.

A macrorregião do Vale do Jequitinhonha está entre as que apresentam piores indicadores sociais do Estado de Minas Gerais ${ }^{14}$. As regiões com piores condições socioeconômicas estão associadas também com as piores condições periodontais em adolescentes brasileiros e de outras partes do mundo. Neste estudo, os resultados não foram diferentes ${ }^{1,7,9-11}$. Estudo de revisão sistemática sugere uma relação inversa entre os indicadores socioeconômicos e os desfechos periodontais por indicadores de renda e escolaridade ${ }^{12}$. Estudos realizados com adolescentes demonstraram forte associação entre sangramento gengival, cálculo dental e variáveis contextuais ${ }^{1,2,9}$. Estudo realizado por Tomita et al. ${ }^{24}$ com 7.700 adolescentes identificou que aqueles com melhores ní- 
veis socioeconômicos estavam mais preocupados com a prevenção de doenças bucais.

Altas prevalências de sangramento gengival e cálculo dental em adolescentes aumentam o risco de desenvolvimento de doença periodontal e, consequentemente, a perda precoce dos dentes e a necessidade de tratamentos complexos no futuro. Contudo, sabe-se que nem todos os sítios com sangramento, mesmo em indivíduos jovens com maior suscetibilidade à doença periodontal, irão desenvolver perda de inserção uma vez que essa enfermidade resulta de uma complexa inter-relação entre agentes infecciosos e fatores ligados ao hospedeiro ${ }^{2,3,6,25,26}$. Nos 450 adolescentes examinados foram encontradas altas prevalências para sangramento gengival, com 51,56\% (CPI = 1), e de cálculo, próximo a 44,88\% (CPI $=2+6)$. A maior variação observada para os adolescentes foi em relação ao componente sadio, apenas 16 $(3,6 \%)$ indivíduos não apresentaram alterações gengivais, sendo que, no inquérito brasileiro SB2010, a população total de adolescentes sadio era $50,9 \%$ e na região sudeste era de $56,8 \% \%^{8,11}$. Esses resultados mostram, no mínimo, que os adolescentes desta região têm uma higiene oral muito deficitária. É um resultado bastante significativo uma vez que isso pode ter muitas causas e é preciso pesquisá-las.

O sextante superior anterior foi o que apresentou menor alteração gengival e pelo menos um estudo também obteve este resultado ${ }^{11}$. Este fato pode estar relacionado com a maior preocupação com a aparência ${ }^{27}$. Com relação à presença de cálculo, o sextante inferior anterior teve a maior frequência, assim como no SB Brasil 2010 ${ }^{11}$. Ambos os resultados podem estar relacionados a deficiências na escovação e no acesso a serviços de saúde bucal por parte dos adolescentes ${ }^{25,26}$. Estudo anterior associou fatores psicossociais de autoestima e autocuidado com maior frequência de escovação e uso de fio dental (fatores internos do indivíduo) ${ }^{27}$. Já o acesso foi considerado um fator externo que não depende exclusivamente do indivíduo e, consequentemente, mais complexo de ser soluciona$\mathrm{do}^{27}$. O cálculo dental não é revomido com hábitos de higiene oral, sendo necessária a presença de profissional. Este estudo sugere a utilização da presença de cálculo dental em adolescentes como marcador de acesso ao serviço de saúde bucal ${ }^{27}$.

A análise dos dados através da Anacor permitiu a formação de 2 grupos: o grupo $1 \mathrm{com}$ adolescentes com periodonto sadio, 19 anos de idade, que trabalhavam e tinham maior renda familiar e o grupo 2, com periodonto apresentando sangramento, com 15 e 16 anos de idade, de ambos os sexos, cursando o ensino fundamental, com menor renda familiar, que se declararam pardos ou negros e não trabalhavam.

Pela Anacor foi possível estabelecer a relação entre gengivite e presença de cálculo com um conjunto de fatores contextuais que podem influenciar o aparecimento da primeira em adolescentes. A técnica também permitiu captar a relação das variáveis estudadas com os dois grupos de indivíduos (sadios e doentes) e ainda as inter-relações entre variáveis. Outros estudos também utilizaram o mesmo tipo de análise, Mota et al. ${ }^{22}$ descreveram o perfil da vítima de violência cometida pelo parceiro utilizando a Anacor, enquanto Rezende et al. ${ }^{23}$ utilizaram também a Análise de Correspondência Múltipla para estudo da mortalidade de idosos com desnutrição.

Os adolescentes do grupo 1, são os mais velhos, estão trabalhando e têm renda familiar maior, propiciando mais acesso aos produtos de higiene bucal e à informação para o autocuidado. Estudos $^{25-27}$ apontam que as razões para que um grupo utilize o serviço odontológico são a percepção da sua importância e o nível de educação em saúde para a formação de comportamento saudável. É importante lembrar que a sociabilização e a preocupação com a estética levam os adolescentes mais velhos a perceberem a necessidade do autocuidado, pois estes estão em um processo adiantado de desenvolvimento da consciência crítica em relação aos mais jovens. Numa sociedade cada vez mais individualista e competitiva, o desejo de possuir uma boa aparência não é mais encarado como sinal de vaidade e sim de necessidade. Em um estudo com adolescentes ${ }^{26}$, $27 \%$ procuraram o serviço odontológico para tratamento ortodôntico, $22 \%$ para consulta de rotina, $12 \%$ por dor ou cárie e $10 \%$ para limpeza/ profilaxia, o que vai ao encontro dessa afirmativa.

$\mathrm{O}$ fato de estarem trabalhando pode ser fator decisivo em suas vidas, podendo ter consequências tanto positivas quanto negativas ao seu desenvolvimento físico e psicossocial, em função da competição que se estabelece entre as atividades de trabalho extraescolar ou domiciliar e as atividades escolares, de esporte e lazer, portanto, aquelas que possibilitam um meio saudável de formação psicológica e social do jovem ${ }^{28}$. Muitos adolescentes que frequentam o ensino público precisam trabalhar para ajudar a compor o orçamento familiar, colocando, muitas vezes, a atividade escolar em segundo plano. Para os adolescentes, o trabalho tem um significado mais profundo, ligado intimamente com a maturidade e a emancipação econômica ${ }^{28}$. 
Nesse sentido, estando trabalhando, o jovem passa a ter mais facilidade de acesso aos bens de consumo e inclusive aos de higiene bucal e é fato que a boa limpeza dos dentes é fator preponderante para a saúde da gengiva ${ }^{26-28}$. Além do mais, pode também ter acesso ao serviço privado de saúde bucal, uma vez que o acesso ao serviço público é mais difícil e, geralmente, funciona no horário comercial que também é o do trabalho ${ }^{27,28}$.

A relação positiva entre a gengivite e os adolescentes do grupo 2 da região estudada pode ser explicada pela não utilização do serviço de saúde bucal, uma vez que observou-se a presença de cálculo dental em 44,88\% (Código CPI $2+6$ ) dos adolescentes, sugerindo o não uso ${ }^{4,6,27}$ (Tabela 2).

As condições socioeconômicas desfavoráveis, presentes no grupo 2, tais como pouca escolaridade e baixa renda, dificultam o acesso às informações sobre saúde e, também, a compra de produtos necessários à higiene bucal, como pasta de dente e fio dental ${ }^{6,12,26}$. Este fato pode favorecer o acúmulo de placa bacteriana e, consequentemente, aumentar o risco de problemas periodontais. Por outro lado, a adolescência é uma fase de transformações físicas, biológicas, psicológicas e comportamentais e não é raro o adolescente negligenciar cuidados com a saúde bucal, reduzindo a escovação dentária, principalmente os mais joven $s^{25}$. A presença de sangramento gengival em adolescentes sugere inadequada higiene oral ${ }^{10,26}$, a qual, para ser bem realizada, inclui a escovação dentária e o uso de fio dental ${ }^{29}$. Em estudo de Flores e Drehmer ${ }^{29}$ foi observada uma resistência ao uso do fio dental por adolescentes devido à dificuldade do seu uso, à habilidade requerida e à preguiça em adotá-lo como rotina. Nesse mesmo estudo, os dentistas foram apontados como os responsáveis pelo ensinamento da escovação. E o sangramento gengival não era percebido como problema que merecesse tratamento ${ }^{29}$.

Na região estudada, $51,56 \%$ dos adolescentes apresentou sangramento gengival, o que pode estar relacionado com os hábitos de higiene oral. Estudo realizado por Davoglio et al. ${ }^{27}$ associou o uso do fio dental e a frequência de escovação a melhores condições socioeconômicas de adolescentes. As mulheres possuem maiores preocupações com a aparência e, portanto, buscam mais os serviços de saúde bucal $^{24}$. Entretanto, pela Anacor, ambos os sexos permaneceram no grupo 2 (doente). Ou seja, a presença da doença, em adolescentes residentes no Vale do Jequitinhonha, pode acometer negativamente esta população. Este fato reforça o contexto multifatorial e hierarquizado para a determinação de hábitos de higiene oral e do estado de adoecimento ${ }^{27}$.

A cor da pele parda foi predominante na região estudada (Tabela 1) e a relação com o sangramento gengival foi positiva juntamente com a população negra. Este fato pode ser explicado pelas significativas diferenças raciais existentes no país e a predominância da cor em determinada região ${ }^{30}$. Entretanto, em outro estudo houve associação entre saúde gengival e cor da pele em adolescentes $^{6}$.

Este estudo mostra que quanto maior a vulnerabilidade social maior a possibilidade de doença. A preocupação aqui deve ser o que o serviço de saúde bucal pode oferecer aos adolescentes, como incluí-los nos programas e como motivá -los para o autocuidado, tendo em mente uma questão bastante importante nessa faixa etária e em localidades pobres é estudar e trabalhar ou, ainda pior, estudar ou trabalhar. Nesse sentido, planejar ações apenas em escolas pode não ser eficaz. De fato, a Política Nacional de Saúde Bucal no seu item ampliação do acesso determina que para o grupo de adolescentes exista a necessidade de organizar fluxos para garantir o atendimento. Vê-se que já existe uma preocupação com esse grupo, que tem sido muito pouco incluído nas ações de saúde bucal, e esse é o desafio. Conhecer as necessidades e a estrutura psicossocial da comunidade em que os adolescentes estão inseridos é essencial, incorporando-os em programas de educação e, dessa forma, permitindo que novas ideias e ações se ajustem, surjam e cresçam nesta realidade.

\section{Considerações finais}

Os resultados apontam para o elevado número de adolescentes com experiência de sangramento gengival e cálculo dental, os quais são aspectos reversíveis da doença periodontal. Sendo a saúde gengival importante para a manutenção da saúde bucal na fase adulta, é necessário pensar em estratégias que possam melhorar a higiene bucal dos adolescentes do Vale do Jequitinhonha.

É preciso entender que a saúde bucal é uma demanda social e que por isso deve ter prioridade nas políticas públicas de saúde. A saúde bucal é essencial para a vida das pessoas, pois é através da boca ou cavidade oral que as pessoas, inclusive a população dos adolescentes, expressam suas necessidades básicas de comunicação, alimentação, estética e relacionamento. 


\section{Colaboradores}

EP Fonseca participou da concepção, análise e interpretação dos dados, redação do artigo e aprovação final do manuscrito. AC Palmier participou da concepção, coleta dos dados, análise e interpretação dos dados, redação do artigo e aprovação final do manuscrito. MHNG Abreu e EF Ferreira participaram da concepção, análise e interpretação dos dados, revisão crítica relevante do conteúdo intelectual e aprovação final do manuscrito. AMD Vargas participou da concepção, análise e interpretação dos dados, revisão crítica relevante do conteúdo intelectual, aprovação final do manuscrito e orientadora do trabalho.

\section{Referências}

1. Ababneh KT, Hwaij ZMFA, Khader YS. Prevalence and risk indicators of gingivitis and periodontitis in a Multi-Centre study in North Jordan: a cross sectional study. BMC Oral Health 2012; 12:1.

2. Gesser HC, Peres MA, Marcenes W. Condições gengivais e periodontais associadas a fatores socioeconômicos. Rev Saude Publica 2001; 35(3):289-293.

3. Iquejiri MH, Zárate-Pereira P. Influência dos aspectos socioeconômicos na incidência da gengivite. Rev. Int. de Periodontia Clínica 2005; 2(6/7):107-114.

4. Bassani D, Lunardelli AN. Condições Periodontais. In: Antunes JLF, Peres MA, organizadores. Epidemiologia da Saúde Bucal. Rio de Janeiro: Guanabara Koogan; 2006. p. 68-78.

5. Kinane DF, Lindhe J. Patogênese da periodontite. In: Lindhe J, organizadores. Tratado de periodontologia clinica e implantodontia oral. Rio de Janeiro: Guanabara Koogan; 1999. p. 127-152.

6. Antunes JLF, Peres MA, Frias AC, Crosato EM, Biazevic MGH. Saúde gengival de adolescentes e a utilização de serviços odontológicos, Estado de São Paulo. Rev Saude Publica 2008; 42(2):191-199.

7. Abrahamsson KH, Kock G, Norderyd O, Romao C, Wennstrom JL. Periodontal conditions in a Swedish city population of adolescents: a cross - section study. Swed Dent J 2006; 30(1):25-34

8. Brasil. Ministério da Saúde (MS). Secretaria de Atenção à Saúde. Departamento de Atenção Básica. Coordenação Nacional de Saúde Bucal. Projeto SB Brasil 2003 condições de saúde bucal da população brasileira 2002 - 2003: Resultados principais. Brasília: MS; 2004.

9. Campus G, Cagetti MG, Senna A, Spano G, Benedicenti S, Sacco G. Differences in oral health among Italian adolescents related to the type of secondary school attended. Oral Health Prev Dent 2009; 7(4):323-330.

10. Rebelo MAB, Lopes MC, Vieira JMR, Parente RCP. Dental cáries and gingivitis among 15 to 19 year-old students in Manaus, AM, Brazil. Braz Oral Res 2009; 23(3):248-254.

11. Brasil. Ministério da Saúde (MS). Secretaria de Atenção à Saúde. Departamento de Atenção Básica. Coordenação Nacional de Saúde Bucal. Projeto SB Brasil 2010 condições de saúde bucal da população brasileira: Resultados principais. Brasília: MS; 2011.

12. Bastos JL, Boing AF, Peres KGA, Antunes JLF, Peres MA. Periodontal outcomes and social, racial and gender inequalities in Brazil: a systematic review of the literature between 1999 and 2008. Cad Saude Publica 2011; 27(Supl. 2):S141-S153.

13. Abreu MHNG. A bioestatística é útil em estudos descritivos? Rev Periodontia 2011; 21(1):7-9.

14. Instituto Brasileiro de Geografia e Estatística (IBGE). Dados sociodemográficos da região do Vale do Jequitinhonha (MG). [acessado $2012 \mathrm{dez} 13$ ]. Disponível em: www.ibge.gov.br

15. Levy P, Lemeshow S. Sampling for health professionals. Belmont: Lifetime learning Publications; 1980.

16. Silva NN. Amostragem probabilística. São Paulo: EDUSP; 1998.

17. Szwarcwald CL, Damacena GN. Amostras complexas em inquéritos populacionais: planejamento e implicações na análise estatística dos dados. Rev Bras Epidemiol 2008; 11(Supl. 1):38-45 
18. Brasil. Ministério da Saúde (MS). Secretaria de Políticas de Saúde. Departamento de Atenção Básica. Área Técnica de Saúde Bucal. Projeto SB2000: condições de saúde bucal da população brasileira no ano 2000: manual do coordenador. Brasília: MS; 2001.

19. UFPEL. Centro de Epidemiologia. Questionário da Coorte de 1982. [acessado 2012 nov 11]. Disponível em: http//www.epidemio-ufpel.org.br/site/content/coorte_1982

20. World Health Organization (WHO). Oral health surveys: basic methods. $4^{\text {th }}$ ed. Geneva: WHO; 1997.

21. Greenacre MJ. Correspondence analysis. Computational Statistics 2010; 2(1):613-619.

22. Mota JC, Vasconcelos AGG, Assis SG. Análise de correspondência como estratégia para descrição do perfil da mulher vítima do parceiro atendida em serviço especializado. Cien Saude Colet 2007; 12(3):799-809.

23. Rezende EM, Sampaio IBM, Ishitani LH, Martins EF, Vilella LCM. Mortalidade de idosos com desnutrição em Belo Horizonte, Minas Gerais, Brasil: uma análise multidimensional sob o enfoque de causas múltiplas de morte. Cad Saude Publica 2010; 26(6):1109-1121.

24. Tomita NE, Pernambuco RA, Lauris JRP, Lopes ES. Educação em saúde bucal: uso de métodos participativos. Rev da Fac de Odont de Bauru 2001; 9(1/2):63-69.

25. 25. Carvalho RWF, Santos CNA, Oliveira CCC, Gonçalves SRJ, Novais SMA, Pereira MAS. Aspectos psicossociais dos adolescentes de Aracaju (SE) relacionados à percepção de saúde bucal. Cien Saude Colet 2011; 16(Supl. 1):1621-1628.

26. Novaes Júnior AB, Souza SL, Taba Junior M, Grisi MF, Suzigan LC, Tunes RS. Control of gingival inflammation in a teenager population using ultrasonic prophylaxis. Braz Dent J 2004; 15(1):41-45.

27. Davoglio RS, Aerts DR, Abegg C, Freddo SL, Monteiro L. fatores associados a hábitos de saúde bucal e utilização de serviços odontológicos entre adolescentes. Cad Saude Publica 2009; 25(3):655-667.

28. Fischer FM, Oliveira DC, Teixeira LR, Teixeira MCTV, Amaral MA. Efeitos do trabalho sobre a saúde de adolescentes. Cien Saude Colet 2003; 8(4):973-984.

29. Flores MTLF, Drehmer TM. Conhecimentos, percepções, comportamentos e representações de saúde e doença bucal dos adolescentes de escolas públicas de dois bairros de Porto Alegre. Cien Saude Colet 2003; 8(3):743-752.

30. Peres MA, Antunes JLF, Boing AF, Peres KG, Bastos JLD. Skin colour is associated with periodontal disease in Brazilian adults: a population-based oral health survey. J Clin Periodontol 2007; 34(3):196-201.

Artigo apresentado em 24/09/2014

Aprovado em 17/04/2015

Versão final apresentada em 19/04/2015 